Ger J Exerc Sport Res 2020 · 50:435-443 https://doi.org/10.1007/s12662-020-00678-3 Received: 8 May 2020

Accepted: 7 September 2020

Published online: 6 October 2020

(c) The Author(s) 2020

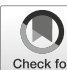

\author{
Jeannine Ohlert ${ }^{1,2,3}$ (D) $\cdot$ Thea Rau' $\cdot$ Bettina Rulofs ${ }^{4} \cdot$ Marc Allroggen $^{1}$ \\ 'Department of Child and Adolescent Psychiatry and Psychotherapy, University Hospital Ulm, Ulm, \\ Germany \\ ${ }^{2}$ German Sport University Cologne, The German Research Centre for Elite Sport—momentum, Cologne, \\ Germany \\ ${ }^{3}$ Institute of Psychology, German Sport University Cologne, Cologne, Germany \\ ${ }^{4}$ Department of Sport Sociology, University of Wuppertal, Wuppertal, Germany
}

\title{
Comparison of elite athletes' sexual violence experiences in and outside sport
}

\section{Background}

\section{Comparison of elite athletes' sexual violence experiences in and outside sport}

Studies indicating that a considerable number of athletes experience sexual violence in their sport are available for a number of countries. A recent study conducted in Germany with an extensive sample of elite athletes shows that more than every third athlete has experienced sexual violence in the field of sport, $11 \%$ even a severe form of sexual violence (Ohlert, Seidler, Rau, Rulofs, \& Allroggen, 2018). Researchers conclude that the sport system itself may bear certain risk factors for the athletes to become victims of sexual violence (Brackenridge, 2001). However, studies directly comparing the sexual violence experiences of athletes in sport with their experiences in the remainder of their life are scarce and showing mixed results. Therefore, it is not clear if the sport system itself is a field of risk or if the athletes as persons (especially elite athletes) are a vulnerable group for sexual violence who might also suffer from sexual violence experiences outside the sport system. The aim of the current study was therefore to compare the prevalence rates for German elite athletes' sexual violence experiences in the field of sport with those outside sport.
A serious problem for the comparison of earlier studies in the area of sexual violence in sport is the fact that almost every publication uses another definition of sexual violence and thus includes more or less incidents of violence into the definition. Within the last few years, however, one of the most commonly used definition in the field of sport is the one used by Alexander, Stafford, and Lewis (2011) who define sexual violence in sport as a "behaviour towards an individual or group that involves sexualised verbal, non-verbal or physical behaviour, whether intended or unintended, legal or illegal, that is based upon an abuse of power and trust and that is considered by the victim or a bystander to be unwanted or coerced" (p. 61). As this is a relatively broad definition of sexual violence including all possible degrees of severity, it was decided to use the definition for the current study. Still, it is important to be able to differentiate between different forms of sexual violence; therefore, Brackenridge (2001) created a continuum of sexual violence in sport, ranging from sexual harassment or 'the chilly climate' to sexual abuse or 'groomed or coerced' with a 'grey zone' ('unwanted attention') in between. Another naming was used by Alexander et al. (2011); they titled the three categories as 'sexual harassment', 'grey zone' and 'sexual harm'. Vertommen and colleagues (Ver- tommen et al., 2016) included also the frequency of occurrence of the single incidents into their categorization of severity, and differentiated between 'mild sexual violence, 'moderate sexual violence' and 'severe sexual violence'. In this manner, a situation of sexual harassment is categorized as 'mild' if it happens only once, but can also be 'severe' if it occurs regularly and/or over a longer period of time (for the detailed assignment to the different categories see Vertommen et al., 2016). In contrast to the other categorization systems, this kind of categorization takes into account the fact that being exposed to sexual harassment over a longer period of time might also lead to serious harm in the affected person (Vertommen et al., 2016). Therefore it was decided to use it for the current study, even though it is of course not without disadvantages.

Numerous international studies are available on the general prevalence of sexual violence, especially in children and adolescents. From two meta-analyses, it is evident that during childhood and adolescence, on average $18 \%$ to $19.2 \%$ of the girls and $7.4 \%$ to $7.6 \%$ of the boys become victims of sexual violence (Pereda, Guilera, Forns, \& Gómez-Benito, 2009; Stoltenborgh, van Ijzendoorn, Euser, \& Bakermans-Kranenburg, 2011). When it comes to adults, data from the $\mathrm{Na}$ tional Intimate Partner and Sexual Violence Survey in the USA revealed that $43.9 \%$ of the women and $23.4 \%$ of the men had experienced a form of sexual 
violence during their lifetimes (Breiding, 2014). However, when comparing worldwide data, it becomes evident that these numbers vary enormously between different countries, from $1.2 \%$ for females in Shanghai up to $62 \%$ for women in Samoa (Decker et al., 2014; GarcíaMoreno, Jansen, Ellsberg, Heise, \& Watts, 2005). A European Study by Krahé and colleagues (Krahé et al., 2015) on young people's sexual victimization in ten European countries showed that between $19.7 \%$ and $52.2 \%$ of the females and between $10.1 \%$ and $55.8 \%$ of the males reported at least one incident of sexual victimization since the age of consent. This large variation is not only to be explained by cultural diversity, but mostly has its reason in different definitions, instruments, samples and/or methodologies used in the different studies.

When looking especially at Germany, a comprehensive survey on the prevalence of sexual abuse in children younger than 16 years (by a person at least 5 years older), which included 11,428 people between the ages of 15 and 40 (51.9\% female), found a prevalence of sexual violence with physical contact of $1.5 \%$ for men and $7.4 \%$ for women (Stadler, Bieneck, \& Pfeiffer, 2012). Focusing on adults, a recent representative study by Allroggen and colleagues (Allroggen et al., 2016) revealed that $1 \%$ of the adults had experienced any form of sexual violence within the last 12 months, $0.6 \%$ of the men and $1.2 \%$ of the women. The prevalence for women in Germany concerning any unwanted sexual actions after the age of 16 is $11.9 \%$ (Bundesministerium für Familie Senioren Frauen und Jugend, 2004); no study is currently available for men's lifetime prevalence of sexual violence experiences in Germany.

Focusing on sexual violence in organized sport, several empirical studies are available from different countries. However, as they also differ vastly according to the used definitions, target groups, perpetrator definitions and countries, diverging prevalence rates were identified. For example $1.9 \%$ of the female college students in an American study reported that their coach had made sexual approaches towards them (Volkwein, Schnell, Sherwood, \& Livezey,
1997), whereas $62.9 \%$ of the student athletes in a Danish study had experienced sexist jokes from the coach (Toftegaard Nielsen, 2001). More recent studies refer mostly on a wider definition of sexual violence (as described before), and do not only target the coach as a perpetrator. In these studies, a prevalence rate of $28 \%$ in Norwegian female national team athletes could be found (Fasting, Brackenridge, \& Sundgot-Borgen, 2004), whereas in Great Britain, $34 \%$ of the female and $17 \%$ of the male athletes were affected by sexual violence (Alexander et al., 2011). The British study used the same definitions and item wordings as the Flemish/Dutch study by Vertommen et al. (2016), but the latter also differentiated between different stages of severity of the sexual violence experiences. In the Netherlands, the prevalence rate for sexual violence in sport was $11.9 \%$, for Flanders it was $16.7 \%$. For international elite athletes, even an increased risk was found with a lifetime prevalence for sexual violence of $28.6 \%(\mathrm{OR}=2.54)$. When looking only at severe sexual violence independent from level of competition, the prevalence rate was $5.5 \%$; $6.5 \%$ for female and $4.4 \%$ for male athletes. On the other hand, a representative study on college athletes in the USA showed that lifetime prevalence for forced sexual intercourse was significantly lower in athletes than in non-athletes (Fasting, Brackenridge, Miller, \& Sabo, 2008). For Germany, the same definition of sexual violence as in the study by Vertommen et al. was used: the basic prevalence rates are depicted in 0 Table 2; prevalence rates for different subgroups are reported elsewhere (Ohlert et al., 2018).

Although there are no studies which compare prevalence rates of sexual violence in different social environments for the same person, a review of reviews by Maniglio (2009) indicates that victims of child sexual abuse are more likely to experience more incidents of sexual abuse in their later life. Thus, instead of focusing on sexual violence that athletes experience in the sport context, it might be more useful to concentrate on the athletes themselves as persons. Until now, however, few studies focusing on the lifetime prevalence of sexual violence espe- cially in elite athletes are available. In a large scale study by Fasting, Brackenridge, and Sundgot-Borgen, $(2003,2004)$ on Norwegian female elite athletes, the respondents also report prevalence rates for their sexual violence experiences outside sport. Within their sample, $28 \%$ of the athletes had been exposed to sexual harassment in sport, but $39 \%$ had experienced sexual harassment outside sport, summing up to $51 \%$ of the athletes with a sexual violence experience independent from the context (Fasting et al., 2004). Furthermore, a comparison with a group of female non-athletes revealed that athletes experienced the same amount of sexual harassment as the nonathletes (Fasting et al., 2003). However, in another study, Parent and colleagues (Parent, Lavoie, Thibodeau, Hébert, \& Blais, 2016) conducted a prevalence study with a representative sample of 6450 children-14 to 17 year olds-in Quebec. For those in the sample considered to be 'athletes' (i.e., affiliated to a sports club), the total prevalence rate for lifetime sexual abuse was $8.8 \%$, whereas only $0.8 \%$ indicated sexual abuse and $0.4 \%$ sexual harassment by the coach (i.e., in the sport context). The same tendency, i.e., a higher risk for sexual violence outside sport was also reported by Leahy and colleagues (Leahy, Pretty, \& Tenenbaum, 2002) for Australian elite athletes, as of those who reported any sexual abuse in their lifetime (29\%), $46 \%$ indicated an incident in sport, but $70 \%$ outside the sport context.

These findings are not in line with earlier considerations and theoretical assumptions that the social structures of the sport culture make it easy for perpetrators to find a victim and not be detected by the social environment (Brackenridge, 2001). Especially unequal gender relations, the focus on the body, the strong dependency on the coach in pursuing performance goals and the general socialization in sport towards risk taking and pushing boundaries, but also sportspecific situations like clothing requirements or locker room/shower situations are frequently named as sport-specific structures to foster sexual violence. In the Norwegian study, the female athletes experienced less sexual harassment in the 
context of sport than outside sports; however, their general lifetime prevalence was comparatively high. Thus, the question arises if the current theoretical considerations with the sport culture as a possible risk for young athletes are sufficient explanations, or if rather the athletes themselves are a vulnerable group concerning sexual violence experiences ${ }^{1}$.

The aim of this study was therefore to compare sexual violence prevalence rates in organized sport and outside sports for elite athletes in Germany. Based on theoretical assumptions and previous studies, it was expected (a) that sexual violence prevalence rates would be different in organized sport compared to outside sport, (b) that female athletes would experience more sexual violence than male athletes, as well in sport as outside sports, and (c) that sexual violence experiences in sport and outside sports would overlap. Furthermore, the total lifetime prevalence rates of sexual violence experiences (in sport and outside sports) for elite athletes in Germany are presented.

\section{Methods}

The data reported in this paper were taken from a larger study on sexual violence in sport in Germany; thus the description in the methods section highly overlaps with the one of a previously published paper on basic prevalence data of sexual violence in sport (Ohlert et al., 2018). However, it was decided not to include the following analyses into the original publication in order to highlight the comparison of the context sport compared with the remainder of the athletes' parts of life. Thus, the publication of Ohlert et al. (2018) focusses on general prevalence data and group comparison of sexual violence in sport, whereas the study presented here adds to the body of knowledge by including the comparison of other areas of life and the analysis of the overlap experiences between sexual violence in different contexts as well as adding a lifetime prevalence for elite athletes.

\footnotetext{
1 The consideration of athletes as vulnerable group does in no case imply that athletes are responsible for the violence happening to them or are required to act against it in any way.
}

\section{Participants}

Participants were 1529 German squad athletes above the age of 16 , representing 128 different sports and 57 different sport organisations. The mean age was 21.6 years $(S D=6.7$ years, range $16-59)$, and the experience in their actual sport ranged from 1 to 50 years $(M=12.1$, $S D=6.3)$. In Germany, elite athletes are categorized into four different squads. The highest level is the A squad, which encompasses national team athletes who represent Germany in international competitions. Not all A squad athletes are professional athletes, but most of them receive some federal funding. The B squad serves as an extension of the A squad, including athletes with future chances for the A squad. Junior athletes are categorized into $C$ squad (highest junior squad) and $\mathrm{D} / \mathrm{C}$ or $\mathrm{D}$ squad. The latter squads are mostly organized on federal state level (Landeskader).

To show the distribution of the participants within the different types of sport, a classification with seven categories according to Schaal et al. (2011) was used: (1) aesthetic sports (e.g., gymnastics, figure skating), (2) contact/combat sports (e.g., judo, karate), (3) team ball sports (e.g., basketball, field hockey), (4) aiming sports (e.g., archery, golf), (5) racing sports (e.g., track and field, cycling), (6) racket sports (e.g., badminton, table tennis), and (7) high-risk sports (e.g., ski jumping, toboggan racing). The distribution of the participants in the different demographic categories is depicted in $\bullet$ Table 1.

\section{Measures}

Sexual violence in organized sport To obtain information about sexual violence experiences in sport, the questions from the Dutch/Flemish study by Vertommen et al. (2016) were used. The questionnaire consists of descriptions of 17 different possible sexual violence situations, ranging from sexual harassment to forced sexual intercourse (see Ohlert et al., 2018 for a detailed description). Respondents were asked to indicate their experiences specifically in organized sport. In Germany, 'organized
Ger J Exerc Sport Res 2020 · 50:435-443 https://doi.org/10.1007/s12662-020-00678-3 (c) The Author(s) 2020

J. Ohlert · T. Rau • B. Rulofs · M. Allroggen Comparison of elite athletes' sexual violence experiences in and outside sport

\section{Abstract}

Background. Research on the prevalence of sexual violence in sport mostly focuses on incidents of sexual violence that happen in the context of sport, but few studies are available that systematically target the lifetime prevalence of sexual violence in the athletes (as persons), combining experiences in sport and outside the sport system. The aim of the current study was thus to compare sexual violence experiences of elite athletes in Germany in sport with those outside sport.

Methods. The study was carried out in cooperation with the German Olympic and Paralympic Committee who contacted their registered elite athletes. In total, 1529 elite athletes took part in an online survey and answered questions regarding their sexual violence experiences first in the context of sport and afterwards outside the sport context.

Results. Results show that $54.2 \%$ of the athletes had experienced some form of sexual violence during their lifetime, $20.6 \%$ even a severe incident of sexual violence. Incidents happened more often outside sport than in sport, but $48 \%$ of the victims were affected in both areas of life, indicating a high overlap of victimization experiences. Conclusion. One recommendation from our results is therefore to make even stronger efforts to protect young athletes from becoming a victim of sexual violence. In case a sport club has a person of trust in the club, it can be an important place for athletes with sexual violence experiences (no matter whether experienced inside or outside sport), so that they are being heard and helped.

\section{Keywords}

Squad athletes - Sexual harassment $\cdot$ Sexual abuse - Lifetime prevalence - Sport culture

sports' refers to nonprofit sports organizations like clubs, sports federations or sport-related boarding schools, not to spontaneous or informal regular sport with friends. The answer had to be given on a four-point scale with "Never", "Once", "Twice to four times" and "Five times and more often". 
Table 1 Summary of the demographic characteristics of the sample

\begin{tabular}{|c|c|c|c|}
\hline & & $n$ & $\begin{array}{l}\text { Share of } \\
\text { participants }\end{array}$ \\
\hline \multirow[t]{2}{*}{ Gender } & Male & 672 & $46 \%$ \\
\hline & Female & 854 & $54 \%$ \\
\hline \multirow[t]{4}{*}{ Age groups } & $16-17$ years & 483 & $27 \%$ \\
\hline & $18-20$ years & 613 & $34 \%$ \\
\hline & $21-30$ years & 564 & $31 \%$ \\
\hline & 31 years and older & 138 & $8 \%$ \\
\hline \multirow[t]{8}{*}{ Sport type } & Racing sports & 507 & $33 \%$ \\
\hline & Team ball sports & 336 & $22 \%$ \\
\hline & High risk sports & 181 & $12 \%$ \\
\hline & Contact/combat sports & 159 & $10 \%$ \\
\hline & Aesthetic sports & 145 & $10 \%$ \\
\hline & Aiming sports & 115 & $8 \%$ \\
\hline & Racket sports & 30 & $2 \%$ \\
\hline & Other & 56 & $4 \%$ \\
\hline \multirow[t]{4}{*}{ Squads } & A squad & 274 & $18 \%$ \\
\hline & B squad & 441 & $29 \%$ \\
\hline & C squad & 553 & $36 \%$ \\
\hline & $D / C$ or $D$ squad & 261 & $17 \%$ \\
\hline \multirow[t]{8}{*}{ Occupational status } & Student (high school) & 570 & $37 \%$ \\
\hline & Student (university) & 372 & $24 \%$ \\
\hline & Sport promotion group & 186 & $12 \%$ \\
\hline & Working full time & 126 & $8 \%$ \\
\hline & Professional athlete & 107 & $7 \%$ \\
\hline & Apprenticeship & 67 & $4 \%$ \\
\hline & Working part time & 44 & $3 \%$ \\
\hline & Other occupation & 57 & $4 \%$ \\
\hline Paralympic athlete & - & 93 & $6 \%$ \\
\hline Migration background & - & 209 & $14 \%$ \\
\hline
\end{tabular}

\section{Sexual violence outside sports In order} to compare sexual violence experiences in and outside sports, the same set of questions and the same answering scale was used to assess sexual violence experiences outside sports. The only difference was that here, respondents had to indicate how often they had experienced the respective situation outside organized sport.

\section{Demographics and other questions}

Furthermore, the participants were asked to answer demographic questions concerning their age, gender, sport, squad membership, duration in their sport, and occupational status.

\section{Procedure}

The study was based on the ethical guidelines of the American Psychological Association and the protocol for cross sectional studies of sexual abuse in sports (Timpka et al., 2015); ethical approval was given by the ethical committee of the involved university hospital. Due to ethical considerations, only athletes with a minimum age of 16 years were included. Athletes were contacted via email which included a link to the online questionnaire. The email was sent by the German Olympic Sports Federation (DOSB), who hosts a list of all German Olympic squad tional Paralympic Committee (DBS) for all 300 Paralympic athletes. As the DOSB and DBS are the two largest umbrella athletes $(N=6699)$ and the German Na- organizations in Germany, the two lists used for this survey should comprise the vast majority of German elite athletes. Athletes were informed that their participation was voluntary and that they could withdraw from their participation anytime. Participants had to give their informed consent in order to start with the survey. The mean duration for answering the whole questionnaire was $17 \mathrm{~min}$. A share of $22 \%$ of the total population answered the questions regarding sexual violence and was thus included in this study.

\section{Analyses}

All data analyses were conducted via SPSS 25 (IBM Corporation, Armonk, NY, USA). A data screening procedure according to Tabachnick and Fidell (2013) was carried out prior to any further analyses. As we have used an online questionnaire for this study and the answers to the respective questions were not mandatory, it has to be noted that the number of answers regarding questions concerning sexual violence outside sport is lower than the total sample because of missing values $(n=1416)$. This reduction in sample size can be explained by the fact that the questions concerning experiences outside sport were placed at the end of the questionnaire.

According to Vertommen et al. (2016), the given situations of sexual violence were differentiated into the three categories of severity: 'mild sexual violence', 'moderate sexual violence' and 'severe sexual violence'. In general, it needs to be recognized that the experience of sexual violence is subjective and depending on individual backgrounds and interpretations. Forms of sexual violence that are labelled as 'mild' in this study might as well be felt as 'severe' by an individual person. Furthermore, it should be noted that a person who was categorized with a 'severe' experience might also have additional 'mild' or 'moderate' experiences, as in this kind of categorization, only the worst experience was counted. When adding up the experiences of sexual violence in sport and outside sports to the lifetime prevalence rate, for each person the most severe 
Table 2 Prevalence rates for sexual violence experiences for the whole sample, in sport and outside sports

\begin{tabular}{|c|c|c|c|c|c|c|}
\hline & \multicolumn{2}{|c|}{ Total sample } & \multicolumn{2}{|l|}{ Male } & \multicolumn{2}{|l|}{ Female } \\
\hline & $\begin{array}{l}\text { In sport } \\
(N=1526)\end{array}$ & $\begin{array}{l}\text { Outside sports } \\
(N=1416)\end{array}$ & $\begin{array}{l}\text { In sport } \\
(n=672)\end{array}$ & $\begin{array}{l}\text { Outside sports } \\
(n=627)\end{array}$ & $\begin{array}{l}\text { In sport } \\
(n=854)\end{array}$ & $\begin{array}{l}\text { Outside sports } \\
(n=789)\end{array}$ \\
\hline Overall sexual violence & $37.2 \%{ }^{\mathrm{a}}$ & $43.4 \%{ }^{\mathrm{a}}$ & $23.8 \%^{b}$ & $30.1 \%^{b}$ & $47.8 \%^{b}$ & $53.9 \%^{b}$ \\
\hline Mild sexual violence & $15.9 \%$ & $15.1 \%$ & $11.2 \%^{b}$ & $13.9 \%$ & $19.6 \%^{\mathrm{b}}$ & $16.1 \%$ \\
\hline Moderate sexual violence & $10.1 \%$ & $11.2 \%$ & $6.0 \%^{b}$ & $5.9 \%^{\mathrm{b}}$ & $13.3 \%^{b}$ & $15.3 \%^{b}$ \\
\hline Severe sexual violence & $11.3 \%^{\mathrm{a}}$ & $17.0 \%{ }^{\mathrm{a}}$ & $6.7 \%^{b}$ & $10.2 \%^{b}$ & $14.9 \%^{b}$ & $22.4 \%^{b}$ \\
\hline
\end{tabular}

experience was counted, independent of the context of the respective incident and of other, less severe experiences.

For the comparison of prevalence rates in sport and outside sports, McNemar Tests were used with odds ratio as effect size. Gender differences were calculated with chi-square tests; the effect size was indicated as Cramer's V. Spearman correlations were applied to analyse the overlap of experiences in sport and outside sports.

\section{Results}

\section{Initial data analyses}

Overall, $37.2 \%$ of the participants indicated that they had experienced one of the predefined situations of sexual violence in the sport setting; $11.3 \%$ had even experienced a severe form of sexual violence. Outside sport, the prevalence rate was at $43.4 \%$ for the whole sample, and $17.0 \%$ had experienced a severe form of sexual violence. All prevalence rates for the current sample are depicted in - Table 2.

\section{Comparison of prevalence rates in sport and outside sports}

For the overall experience of sexual violence in sport and outside sport, the McNemar test revealed a significant difference between the two situations $\left(\chi^{2}(1)=43.67 ; p<0.001 ; O R=1.41\right)$ with the prevalence being higher outside sports than in sport (see also - Table 2). For so-called mild sexual violence, the prevalence rate did not differ significantly in sport and outside sports $\left(\chi^{2}(1)=0.13\right.$; $p=0.723$ ), and the same holds true for moderate forms of sexual violence $\left(\chi^{2}(1)=2.43 ; p=0.119\right)$. For severe forms, however, the prevalence rate was significantly higher outside sports than in the sport context $\left(\chi^{2}(1)=47.51 ; p<0.001\right.$; $O R=2.13)$.

\section{Gender comparison}

On the overall level, the comparison between male athletes and female athletes revealed a significant difference in sexual violence prevalence, as well in sport $\left(\chi_{(1)}^{2}=91.32, p<0.001, V=0.25\right)$ as outside sports $\left(\chi^{2}{ }_{(1)}=80.05, p<0.001\right.$, $V=0.24$ ), with females reporting higher prevalence rates than males (see also - Table 2). The same pattern can be found for almost all forms of sexual violence, as females reported significantly higher prevalence rates for moderate and severe sexual violence, both in sport (moderate: $\chi^{2}(1)=22.68, p<0.001$, $V=0.12$; severe: $\chi^{2}(1)=25.13, p<0.001$, $V=0.13$ ) and outside sports (moderate: $\chi^{2}(1)=31.37, p<0.001, V=0.15$; severe: $\left.\chi^{2}(1)=36.98, p<0.001, V=0.16\right)$. Only for so-called mild sexual violence, the prevalence rates between the two groups did not differ outside sports $\left(\chi_{(1)}^{2}=1.34\right.$, $p=0.247$ ); in sport, however, the prevalence rate was higher for female athletes $\left(\chi^{2}(1)=19.86, p<0.001, V=0.11\right)$.

\section{Total prevalence of sexual violence} experiences in elite athletes, and overlap of sexual violence experiences in sport and outside sports

When combining the experiences of sexual violence in sport and outside sport to a total prevalence rate of sexual violence experiences in elite athletes, results showed that $54.2 \%$ of the athletes had experienced at least one form of sexual violence at least once during their life- time. Almost one fifth (18.6\%) of all athletes had experienced a so-called mild form of sexual violence, $13.9 \%$ a moderate form, and more than one in five athletes $(20.6 \%)$ were exposed to a severe form of sexual violence during their lifetime (either in sport or outside sport). Also for this result, significant gender differences were evident $\left(\chi^{2}(3)=121.25\right.$, $p<0.001, V=0.28$ ), with $40.9 \%$ of the male and $66.3 \%$ of the female athletes reporting at least one incident of sexual violence during their lifetime (independent from context). In total, $18.2 \%$ of the male athletes had experienced a socalled mild form of sexual violence, $8.6 \%$ a moderate form, and $14.1 \%$ a severe form. For the females, $19.2 \%$ indicated a mild form, $18.2 \%$ a moderate form, and $28.9 \%$ a severe form of sexual violence.

- Table 3 shows the overlap of sexual violence experiences in sport and outside sports. It is evident that almost half of the athletes with sexual violence experiences $(48.0 \%)$ were affected in both fields of their life, $34.9 \%$ only outside sports, and $17.1 \%$ only inside sports. When separating according to severity, the overlap increases from $27.0 \%$ in mild sexual violence up to $64.9 \%$ in severe sexual violence (- Table 3). This is in accordance with the correlation of both experiences which is of a moderate effect size with $\rho=0.44(p<0.001)$ when using an overall perspective (sexual violence yes/no). When taking into account the different levels of severity, the correlation is approximately at the samelevel with $\rho=0.47$ $(p<0.001)$, almost reaching a large effect size. Gender also has an effect on this correlation, as for male athletes, the correlation between sexual violence experiences in sport (yes/no), and outside sports (yes/no) is at $\rho=0.34(p<0.001)$, whereas for female athletes, it rises to 
Table 3 Percentage of athletes who have experienced the respective form of sexual violence

Only in sport

Only outside sports

In both fields

Overall sexual violence $(n=739)$

$17.1 \%$

$34.9 \%$

$48.0 \%$

Mild sexual violence $(n=248)$

$28.2 \%$

$44.8 \%$

$27.0 \%$

Moderate sexual violence

$15.6 \%$

$35.4 \%$

$49.0 \%$

$(n=192)$

Severe sexual violence $(n=299) \quad 8.7 \%$

$26.4 \%$

$64.9 \%$

$\rho=0.49(p<0.001)$. The same pattern can be noted if level of severity is taken into account, with $\rho=0.32(p<0.001)$ for the male athletes and $\rho=0.45(p<0.001)$ for the female athletes.

\section{Discussion}

Most studies on sexual violence in sport focus on sport as a context to foster sexual violence (Alexander et al., 2011; Fasting et al., 2004; Toftegaard Nielsen, 2001; Vertommen et al., 2016; Volkwein et al., 1997), and not on the athletes as vulnerable persons. Thus, our study is one of the first to report lifetime prevalence rates of sexual violence experiences for elite athletes. Besides that, the aim of the study was to show differences between sexual violence experiences in sport and outside sports, gender differences, but also the correlation between sexual violence experiences in sport and outside sport. Our data reveal that prevalence rates for sexual violence experiences outside sport are higher than in the sport context. This especially applies to severe forms of sexual violence. According to our expectations, females report to be more exposed to sexual violence than males, independent from context and level of severity, and sexual violence experiences in sport and outside sport highly overlap, especially for severe forms of sexual violence. Furthermore, our study shows that the overall lifetime prevalence of sexual violence in athletes is relatively high when compared to studies of the general population.

Different aspects from our results are remarkable; first of all the fact that prevalence rates of athletes' sexual violence experiences outside sport exceed the ones from the sport context, and especially severe sexual violence is significantly elevated. This is contrary to the assumptions derived from qualitative studies and the- oretical considerations of several particular risk factors in sport (Brackenridge, 2001), but in line with the findings of other quantitative studies (Fasting et al., 2004; Leahy et al., 2002; Parent et al., 2016) that also revealed higher prevalence rates outside sports than in the sport context. At first glance, these results challenge the previous theoretical considerations about the sport system being a field with specific risk factors for sexual violence against athletes. However, it should be kept in mind that qualitative studies as well as theoretical approaches do not focus on the comparison between sport and other contexts of life, but rather exclusively on the sport context. Derived from the reports of affected persons, it is not surprising that from their point of view, the sport culture appears a culture of risk. However, given also the results of the other quantitative studies in this area, our findings should be regarded in a differentiated way:

Prevalence rates for sexual victimization in and outside sport among athletes appear high when compared to population based studies with young people using a broad definition of sexual violence (Averdijk, Mueller-Johnson, \& Eisner, 2012). It seems that elite athletes are a highly vulnerable group for sexual violence experiences, given that four in ten male athletes and even two out of three female athletes reported at least one sexual violence event during their lifetime (at an average age of 21 years!), and more than one in four female athletes were exposed to severe sexual violence. This leads to the question why this is the case. Two different explanations might be possible. The first explanation relates more to the contextual factors of the sport environment, as the highly performanceoriented competitive sport expects from the athletes to always go beyond their initial limits to reach peak performance
(Brackenridge, 2001). Therefore, the athletes are used to discipline their body and to do things with the body they do not feel comfortable with. Through this socialization in sport, they might also comply with unusual requests concerning their sexuality and might have lost the sense for their personal boundaries. The second possible explanation relates more to personal factors of single athletes, for example that those persons who have experienced sexual violence outside sports deliberately turn to sports, and especially elite sports as an addition to or replacement for psychotherapy. Perhaps the field of elite sports offers possibilities to regain control over their life and their bodies, providing time away from home, with a systematic structure and physical requirements that might give the victims of sexual violence the chance to be away from their perpetrator, to structure their life and to receive control over their body in training and competitions. Which of these explanations (a combination of both contextual and personal factors, or others?) is best suited to explain the result can however not be answered by our study, as the necessary details from the respective backgrounds of the athletes were not recorded in this survey. This has to be investigated in further studies.

Our results also provide evidence that sexual violence experiences in sport and outside sports are highly overlapping: half of the athletes who reported at least one event of sexual violence in sport also indicated sexual violence in other areas of life. For victims of severe sexual violence, and also for female athletes, the overlap is even higher. The reason for this phenomenon, however, cannot be given by our data, even though two different explanations are subject to discussion. First of all, if a person is socialized in an abusive surrounding and is for example regularly exposed to sexual violence at home, this person did not have the chance to learn an appropriate way of handling a sexual relationship and might be in search of a close relationship outside their home. This explanation is supported by the review of reviews by Maniglio (2009). Therefore, the former victim might make an easy target for potential offenders in sport, as 
these are always in search of vulnerable persons (Cense \& Brackenridge, 2001). This also fits with the fact that athletes with severe sexual violence experiences are more likely to report overlaps in sexual violence experiences between the two contexts. Another possible explanation is the fact that the German sport club system is build up in a different way than other countries, because sports clubs do not belong to universities or colleges, but are mostly independent clubs that are financed by fees of their members. Many clubs are run by volunteers, based on trust and close relationships. Quite frequently, coaches-especially in the work with adolescents and children-are fathers, mothers, relatives or close friends of the families. Connecting this aspect to sexual violence, it becomes plausible that an abusive father, friend or uncle might also be the coach or the president of the club. Thus, assaults of the same person can happen in the context of sport, but also outside sports and this constellation might have created a high overlap of experiences in our study results. However, future studies are necessary to bring more light into this field and to find out if our explanations are correct.

In accordance with our hypothesis, females reported experiencing sexual victimization more often than males in the context of sport. However, Parent et al. (2016) could not find gender differences concerning sexual abuse by a coach, but the prevalence of reported perceived consensual sexual contacts with a coach was higher than the reported prevalence of abuse. It is possible that some victims explain sexual contacts with coaches as consensual rather than as sexual abuse. By comparing results from different studies, it has to be kept in mind that asking for the perception of a situation may lead to different results than just asking if a situation has happened. Furthermore, it has to be noted that men are in general less likely to report sexual violence than women (McLean, 2013); thus, the prevalence rate for the male athletes might be underestimated.

Several limitations appear in our study. First of all, even though the contact lists from the two largest German sport organization were used, these lists do not contain all squad athletes in Germany, and not all information is included for every athlete-depending on the information provided by the athletes' respective federations. Squad athletes younger than 16 years were not included in the study because of data privacy protection and ethical reasons. Thus, especially those types of sports with a high number of squad athletes below the age of 16 (e.g., gymnastics or figure skating) are underrepresented in our study. Therefore, our results should not be transferred to all kinds of sports, even though our study comprises by far the most extensive sample of elite athletes

Hier steht eine Anzeige. 
that has ever been questioned regarding sexual violence experiences in sport in Germany (and other countries). In addition, as we have only questioned elite athletes, our results are not transferable to recreational sports.

Furthermore, it should be taken into account that our study contained a very sensitive topic and was carried out as an online survey. Therefore, it is possible that athletes who have been affected by sexual violence did not want to participate in the study, either because they did not trust the data privacy protection or because they did not want to actively remember their possibly traumatic experiences. On the other hand it is also plausible that especially this group of athletes was highly motivated to take the survey because they wanted to share their experiences and support prevention against sexual violence for other athletes.

Lastly, it has to be noted that very probably several athletes who were affected by sexual violence in sport have already quit the (elite) sport system because of their experiences; or they might be in a sexual relationship with another person, they are dependent of, and not realise that they have been forced into this relationship until a later time (Longman, 1999). With regard to these cases, the prevalence rates would be underestimated.

\section{Perspective}

To conclude, our study is one of the first to explore sexual violence experiences in athletes not only in the context of sport, but also in other areas of life. This is contrary to most other studies on sexual violence in sport as they focus on sport as a context to foster sexual violence (Alexander et al., 2011; Fasting et al., 2004; Toftegaard Nielsen, 2001; Vertommen et al., 2016; Volkwein et al., 1997). As can be seen from our data-and in line with the data provided by Fasting et al. (2004)-elite athletes seem to be a vulnerable group when it comes to sexual violence experiences. Further studies need to explore if the explanation lies in the culture of organized sport (Brackenridge, 2001) or more in the personal history of the respective athletes (Cense
\& Brackenridge, 2001). Nevertheless, an important consequence from our results is therefore to make even stronger efforts to protect young athletes from becoming a victim of sexual violence. Following the results of our study, the sport clubs seem to be a very important place to offer athletes with sexual violence experiences (no matter whether experienced inside or outside sport) a safe setting and the chance to find a person of trust in their own club, so they can be sure that they are being heard and helped.

\section{Corresponding address}

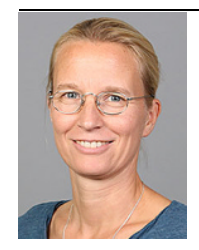

Dr. Jeannine Ohlert

Department of Child and Adolescent Psychiatry and Psychotherapy, University Hospital Ulm

Steinhoevelstr. 5, 89075 Ulm, Germany

j.ohlert@dshs-koeln.de

Funding. This study was supported by the Federal Ministry of Education and Research (Grant FKZ 01SR $1401 \mathrm{XY}$ ). The funding body did not exert any influence in the design of the study, collection, analysis, interpretation of data, nor in writing the manuscript.

Funding. Open Access funding enabled and organized by Projekt DEAL.

\section{Compliance with ethical guidelines}

Conflict of interest. J. Ohlert, T. Rau, B. Rulofs and M. Allroggen declare that they have no competing interests.

All studies performed were in accordance with the ethical standards indicated in each case.

Open Access. This article is licensed under a Creative Commons Attribution 4.0 International License, which permits use, sharing, adaptation, distribution and reproduction in any medium or format, as long as you give appropriate credit to the original author(s) and the source, provide a link to the Creative Commons licence, and indicate if changes were made. The images or other third party material in this article are included in the article's Creative Commons licence, unless indicated otherwise in a credit line to the material. If material is not included in the article's Creative Commons licence and your intended use is not permitted by statutory regulation or exceeds the permitted use, you will need to obtain permission directly from the copyright holder. To view a copy of this licence, visit http://creativecommons.org/licenses/by/4.0/.

\section{References}

Alexander, K., Stafford, A., \& Lewis, R. (2011). The experiences of children participating in organised sport in the UK. Edinburgh: Dunedin Academic Press.

Allroggen, M., Rassenhofer, M., Witt, A., Plener, P.L., Brähler, E., \& Fegert, J. M. (2016). The prevalence of sexual violence: results from a populationbased sample. Deutsches Aerzteblatt International, 113(7), 107-113. https://doi.org/10. 3238/arztebl.2016.0107.

Averdijk, M., Mueller-Johnson, K., \& Eisner, M.P. (2012). Sexual victimization of children and adolescents in Switzerland. Zuerich: UBS Optimus Foundation.

Brackenridge, C. (2001). Spoilsports: understanding and preventing sexual exploitation in sport. London: Routledge.

Breiding, M. J. (2014). Prevalence and characteristics of sexual violence, stalking, and intimate partner violence victimization-National Intimate Partner and Sexual Violence Survey, United States, 2011. Morbidity and Mortality Weekly Report. Surveillance Summaries, 63(8), 1-18.

Bundesministerium für Familie Senioren Frauen und Jugend (2004). Lebenssituation, Sicherheit und Gesundheit von Frauen in Deutschland [Life situation, security, and health of women in Germany]. https://www.bmfsfj.de/blob/ jump/84328/langfassung-studie-frauen-teileins-data.pdf. Last access: 30.09.2020.

Cense, M., \& Brackenridge, C. (2001). Temporal and developmental riskfactors for sexual harassment and abuse in sport. European Physical Education Review, 7(1),61-79.

Decker, M. R., Peitzmeier, S., Olumide, A., Acharya, R., Ojengbede, O., Covarrubias, L., Brahmbhatt, $H_{\text {., }}$ et al. (2014). Prevalence and health impact of intimate partner violence and non-partner sexual violence among female adolescents aged 15-19 years in vulnerable urban environments: A multi-country study. Journal of Adolescent Health, 55(6), S58-S67.

Fasting, K., Brackenridge, C., \& Sundgot-Borgen, J. (2003). Experiences of sexual harassment and abuse among Norwegian elite female athletes and nonathletes. Research Quarterly for Exercise and Sport, 74(1), 84-97. https://doi.org/10. 1080/02701367.2003.10609067.

Fasting, K., Brackenridge, C., \& Sundgot-Borgen, J. (2004). Prevalence of sexual harassment among Norwegian female elite athletes in relation to sport type. International Review for the Sociology of Sport, 39(4), 373-386. https://doi.org/10. $1177 / 1012690204049804$

Fasting, K., Brackenridge, C. H., Miller, K. E., \& Sabo, D. (2008). Participation in college sports and protection from sexual victimization. International Journal of Sport and Exercise Psychology, 6(4), 427-441. https://doi.org/10.1080/1612197X. 2008.9671883

García-Moreno, C., Jansen, H. A. F.M., Ellsberg, M., Heise, L., \& Watts, C. (2005). WHO multi-country study on women's health and domestic violence against women. Geneva: WHO.

Krahé, B., Berger, A., Vanwesenbeeck, I., Bianchi, G., Chliaoutakis, J., Fernández-Fuertes, A., Zygadlo, A., et al. (2015). Prevalence and correlates of young people's sexual aggression perpetration and victimisation in 10 European countries: a multi-level analysis. Culture, Health \& Sexuality, 17, 682-699. https://doi.org/10. 1080/13691058.2014.989265. 
Leahy, T., Pretty, G., \& Tenenbaum, G. (2002). Prevalence of sexual abuse in organised competitive sport in Australia. The Journal of Sexual Aggression, 8(2), 16-36. https://doi.org/ $10.1080 / 13552600208413337$.

Longman, J. (1999). Topfigure-skating coach is accused of sexual misconduct. https://www.nytimes. com/1999/04/11/sports/figure-skating-topfigure-skating-coach-is-accused-of-sexualmisconduct.html. Last access: 30.09 .2020

Maniglio, R. (2009). The impact of child sexual abuse on health: a systematic review of reviews. Clinical psychology review, 29(7), 647-657. https://doi. org/10.1016/j.cpr.2009.08.003.

McLean, I. A. (2013). The male victim of sexual assault. Best Practice \& Research-Clinical Obstetrics \& Gynaecology, 27(1), 39-46. https://doi.org/10. 1016/j.bpobgyn.2012.08.006.

Ohlert, J., Seidler, C., Rau, T., Rulofs, B., \& Allroggen, M. (2018). Sexual violence in organized sport in Germany. German Journal of Exercise and Sport Research, 48(1), 59-68. https://doi.org/10.1007/ s12662-017-0485-9.

Parent, S., Lavoie, F., Thibodeau, M.-Ė., Hébert, M., \& Blais, M. (2016). Sexual violence experienced in the sport context by a representative sample of Quebec adolescents. Journal of Interpersonal Violence, 31(16), 2666-2686. https://doi.org/10. $1177 / 0886260515580366$.

Pereda, N., Guilera, G., Forns, M., \& Gómez-Benito, J. (2009). The prevalence of child sexual abuse in community and student samples: a meta-analysis. Clinical psychology review, 29(4), 328-338.

Schaal, K., Tafflet, M., Nassif, H., Thibault, V., Pichard, C., Alcotte, M., Guillet, T., El Helou, N., Berthelot, G., Simon, S., \& Toussaint, J. F. (2011). Psychological balance in high level athletes: gender-based differences and sport-specific patterns. PloS One, 6(5), 1-9. https://doi.org/10.1371/journal.pone. 0019007.

Stadler, L., Bieneck, S., \& Pfeiffer, C. (2012). Repräsentativbefragung sexueller Missbrauch 2011 [Representative survey sexual abuse 2011]. Hannover: Kriminologisches Forschungsinstitut Niedersachsen.

Stoltenborgh, M., van ljzendoorn, M. H., Euser, E. M., \& Bakermans-Kranenburg, M.J. (2011). A global perspective on child sexual abuse: metaanalysis of prevalence around the world. Child maltreatment, 16(2), 79-101. https://doi.org/10. $1177 / 1077559511403920$

Tabachnick, B.G., \& Fidell, L.S. (2013). Using multivariate statistics. Vol. 6. London: Pearson

Timpka, T., Janson, S., Jacobsson, J., Ekberg, J., Dahlström, Ö., Kowalski, J., Svendin, C. G., et al. (2015). Protocol design for large-scale crosssectional studies of sexual abuse and associated factors in individual sports: Feasibility study in Swedish athletics. Journal of Sports Science and Medicine, 14, 179-187.

Toftegaard Nielsen, J. (2001). THE FORBIDDEN ZONE:Intimacy, sexual relations and misconduct in the relationship between coaches and athletes. International Review for the Sociology of Sport, 36, 165-182. https://doi.org/10.1177/ 101269001036002003.

Vertommen, T., Schipper-van Veldhoven, N., Wouters, K., Kampen, J.K., Brackenridge, C.H., Rhind, D.J.A., van den Eede, F., et al. (2016) Interpersonal violence against children in sport in the Netherlands and Belgium. Child Abuse \&
Neglect, 51, 223-236. https://doi.org/10.1016/j. chiabu.2015.10.006.

Volkwein, K.A.E., Schnell, F.I., Sherwood, D., \& Livezey, A. (1997). Sexual harassment in sportperceptions and experiences of american female student-athletes. International Review for the Sociology of Sport, 32(3), 283-295. 